\title{
Reducing Energy Consumption with Dvfs for Real-Time Services in Cloud Computing
}

\author{
${ }^{1}$ Vrunda J. Patel, ${ }^{2}$ Prof. Hitesh A. Bheda \\ ${ }^{1}$ Researcher of M.Tech Scholar, Department of Computer Engineering RK University, India \\ ${ }^{2}$ Department of Computer Engineering RK University, India
}

\begin{abstract}
Cloud computing has revolutionized the information technology industry by enabling elastic on demand provisioning of computing resources. The increase of Cloud computing has resulted in the organization of large-scale data centers around the world contain thousands of total nodes. This paper presents overview of cloud computing, issues in cloud computing, novel techniques, and software for distributed dynamic consolidation of Virtual Machines (VMs) in Cloud data centers. The goal is to improve the utilization of computing resources and reduce energy consumption under workload independent quality of service constraints. Dynamic VM consolidation leverages fine-grained fluctuations in the application workloads and continuously reallocates VMs using live migration to minimize the number of active physical nodes. Energy consumption is reduced by dynamically deactivating and reactivating physical nodes to meet the current resource demand. The proposed approach is distributed, scalable, and efficient in managing the energyperformance trade-off. In this paper, we introduce DVFS concept with live migration technique to improve efficiency of energy management and adaption scheme on real-time services.
\end{abstract}

Keyword: Cloud Computing, Data Center, Energy Management, Virtualization, Real-time Service, DVFS, Live Migration

\section{Introduction}

Cloud computing recently received considerable attention, as a promising approach for delivering Information and Communication Technologies (ICT) services [1],[2]. Cloud computing is becoming an important opportunities for industry to provide a high degree of scalability and serviceability of computing resources. By definition of cloud and capability to support computing[2] in the different services types such as Iaas Paas or Saas makes cloud computing becomes a favorite computing system today. Computing resources that are available on demand, elimination of an up-front commitment by cloud users and ability to pay per use of computing resources are benefits of cloud computing. These features motivate service providers to increase their resources in datacenter [7] to support user demand that is enlarging significantly such as processor unit, storages and etc. Consequently, electrical power is consumed increasingly and becomes a major concern [8].With more and more suppliers began offering cloud computing services, these services are convenient to users but consuming a lot of energy. Thus, how to save the energy of the data center without affecting both the economic efficiency and system performance is an important issue. Virtualization technology can simulate a variety of different platforms and manage the resources of the system. By applying the virtualization technology, in accordance with the requirements of the users to configure a virtual machine, both the computing environment and resource management problems can be solved.

In cloud computing environment, the amount of workload will affect the loading of the physical machine of cloud server. If in accordance with the appropriate voltage regulation would be effective in improving energy efficiency and reduce the overhead. This paper proposes a mechanism to adjust the system voltage based on the CPU utilization, and migrating tasks in a heavy loaded machine to idle machines [9]. Dynamic CPU allocation with appropriate utilization levels will help service provider to conserve more energy and get better performance. Thus the new technique not only to reduce energy consumption[10] in cloud data center but also to provide better performance by introducing a CPU re-allocation algorithm that combined DVFS concept with live migration technique in three characteristics including Longest Completion Time(LCT), Highest Utilization (HU) and Lowest Utilization (LU).

This paper is prearranged as following. In the Section 2 describes some Overview of Issues in Energy Management, Section III Improving Energy Management with combined concept of DVFS adjustment and Live Migration Mechanism and Section IV we focus Experimental Results /Improvement and Section V presents conclusion. 


\section{Overview of Issues In Energy Management}

\subsection{Definition}

Energy Management has now become one of the major design constraints for current and future cloud data center operators [3],[4]. Rapid growth of the demand for computational power by scientific, business and web-applications has led to the creation of large-scale data centers [5],[6].

\subsection{Energy Management Issues}

Improving energy efficiency is another major issue in cloud computing. It has been estimated that the cost of powering and cooling accounts for 53\% of the total operational expenditure of data centers [11]. In 2006, data centers in the US consumed more than $1.5 \%$ of the total energy generated in that year, and the percentage is projected to grow $18 \%$ annually [4]. Hence infrastructure providers are under enormous pressure to reduce energy consumption. The goal is not only to cut down energy cost in data centers, but also to meet government regulations and environmental standards. Designing energy-efficient data centers has recently received considerable attention. This problem can be approached from several directions. For example, energy efficient hardware architecture that enables slowing down CPU speeds and turning off partial hardware components has become commonplace Energy aware job scheduling and server consolidation are two other ways to reduce power consumption by turning off unused machines. Recent research has also begun to study energy-efficient network protocols and infrastructures. A key challenge in all the above methods is to achieve a good trade-off between energy savings and application performance. In this respect, few researchers have recently started to investigate coordinated solutions for performance and power management in a dynamic cloud environment.

\section{Improving Energy Management Issues With Combined Concept Of Dvfs And Live Migration Mechnism}

\subsection{DVFS (Dynamic Voltage Frequency Scaling)}

As [12],[13] DVFS technique can be applied by monitoring the CPU utilization. When the workload is heavy, real-time migration can be provided for achieving more effective usage of resources under the user unaware situation. Dynamic voltage frequency scaling is a hardware technology that can dynamically adjust the voltage and frequency of the processor in execution time. By applying DVFS technology, without having to restart the power supply, system voltage and frequency can be adjusted in accordance with the specification of the original CPU design into a different working voltage. While CPU works in lower voltage, the energy consumption can effectively be saved.

The power consumption of the CPU is measured by multiplying the voltage square with the system frequency. Where V is the voltage, $\mathrm{F}$ is the frequency, and $\mathrm{C}$ is the capacitive load of the system. The DVFS is the power saving technology by reducing the voltage supply [14]. The reduction of CPU frequency means that the voltage can also be dropped, though it will result in the degradation of the system performance and lead to prolong the execution time. In addition, the overhead of the voltage adjusting should also be considered.

The purpose of the DVFS is to allow the filth of the execution speed of a task by decreasing the CPU frequency and voltage to reduce the power burning up. This technology is often used in real-time systems.

$$
P=V^{Z} \times F \times C
$$

As mentioned above, by applying the DVFS technology, CPU voltage can be lowered, but the execution speed of the task will be reduced. From Equation, we can see that if only reducing the frequency, energy cannot be saved effectively. In the system, $\mathrm{C}$ is the capacitive load of the system, only in lowering the frequency and also reducing the voltage, the power consumption can be saved effectively.

As implemented CloudSim [15] MIPS and frequency are directly related together. They presented three schemes result in reduce energy consumption, Lowest DVFS, Advanced DVFS and Adaptive DVFS, to allocate appropriate MIPS rate to real-time service[16].

\subsection{Combined concept of DVFS adjustment and Live Migration Mechanism \\ 3.2.1. DVFS adjustment mechanism}

A mechanism by applying CloudSim to simulate a large scale cloud data center for energy saving has been proposed in this chapter. The system consists of three parts: the CPU utilization monitoring, DVFS adjustment, and real-time migration. CPU utilization on each host is monitored in the system. According to the measured CPU utilization, an appropriate process will be performed for saving energy consumption.

$$
\begin{gathered}
\text { Vcpui }=\frac{V M}{H O S T} \frac{M I P S i}{M I P S i} \\
\text { CPUutilization }=\frac{\sum_{i=1}^{n} \text { Vcpui }}{H O S T . M I P S i}
\end{gathered}
$$


In CloudSim [17],[18], MIPS (million instructions per second) is used to present the capacity of the host machine, the capacity of VM, and the workload requested by the user. Each workload will be distributed to VMs on different hosts. VMMIPS is the amount of MIPS required for the VM and Host MIPS presents the amount of MIPS the host can support. The utilization of the virtual CPU of a virtual machine, Vcpu, can be calculated by Eq. 1, and the average utilization of CPUs can be calculated by Eq. 2

To reduce energy consumption, we can lower the frequency but inappropriate frequency assigned to virtual machine may result in performance degradation and missing application deadline. implemented CloudSim to show that MIPS and frequency are directly relate presented three schemes result in reduce en Lowest-DVFS, $\partial-$ Advanced-DVFS and Adaptive DVFS, to allocate appropriate MIPS rate to real-time services.

Apart from controlling energy consumption by load balancing or voltage adjustment at current executed job, process live migration technique when host is more overload than dynamic threshold of current time.

\subsubsection{DVFS technique with Live Migration}

As experiment in [16], expressed a better result of energy reduction when implemented DVFS technique with live migration [20] without performance consideration. By this idea, we propose an algorithm that also concerned about upgrading performance and energy conservation for every time interval in three characteristics, Longest Completion Time (LCT), Highest Utilization (HU) and Lowest Utilization (LU).

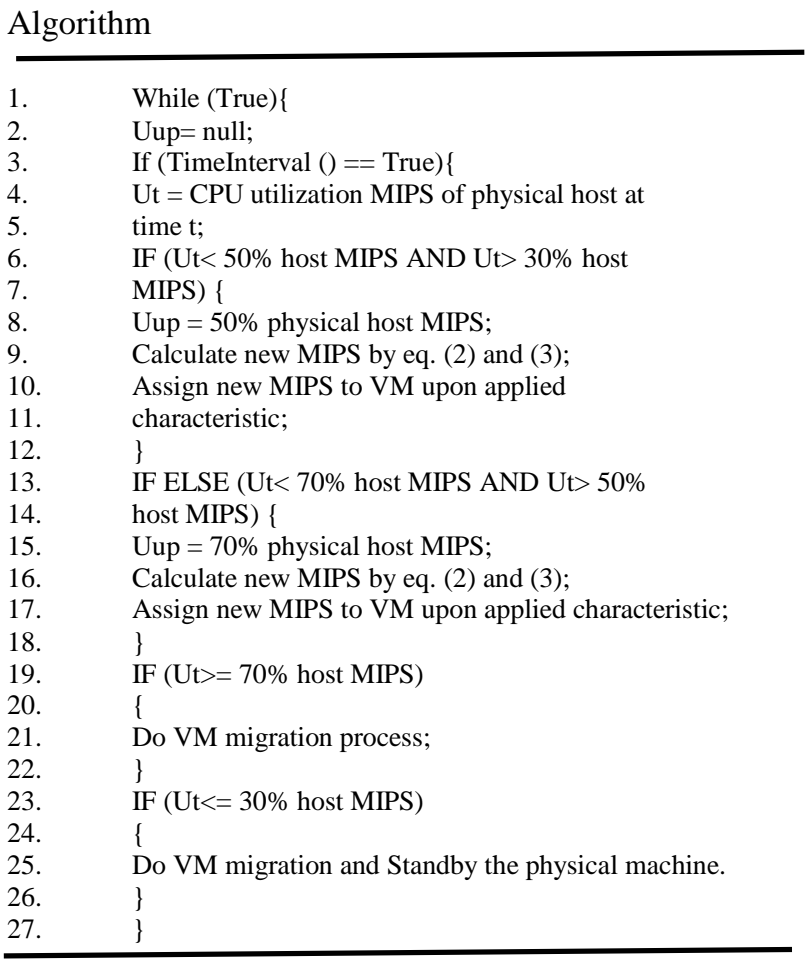

From this proposed algorithm we can use migration technique for better conserving energy. DVFS technique can reduce energy consumption but migration technique is better in power saving.

\section{Experimrnt Result}

The experiment is done on Cloud Sim 3.0.1 by simulated in homogenous environment CPU Concerned.

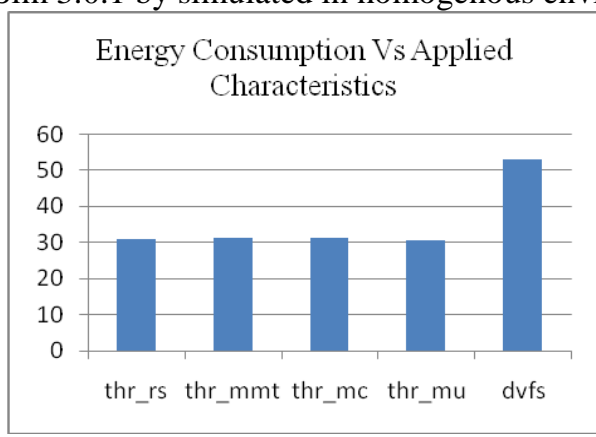

Fig 1: Energy consumption vs applied characteristics 
Fig1 shows the result of energy consumption of proposed techniques (HU, LU, LCT) comparing with the existing techniques (DFVS, 70-30THRMU) in every $300 \mathrm{sec}$ with 100 virtual machines time interval.

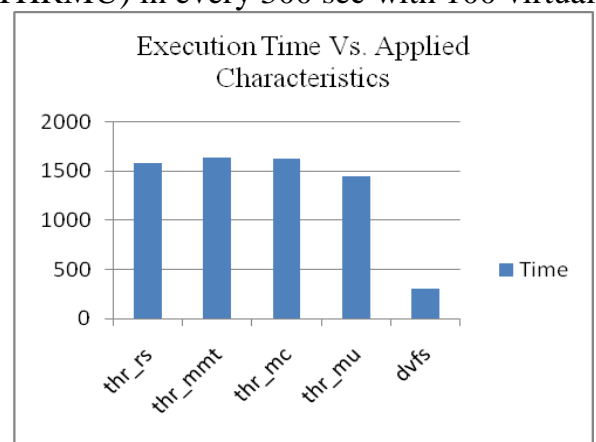

Fig 2: Execution time vs applied characteristics

Fig2 shows the result of Execution time of proposed techniques. It also shows that proposed techniques having less execution time. Both of figures show better results in energy and performance perspective.

\section{Conclusion}

Energy management has become an important issue in computing environments. In this paper we conclude that a dynamic resource management with energy-saving mechanism in the cloud computing environment to reduce the energy consumption. DVFS technique can be applied by monitoring the CPU utilization. When the workload is heavy, real-time migration can be provided for achieving more effective usage of resources under the user unaware situation. The results show the reduction of execution time and energy consumption in various characteristics upon the scheme usage. From the experiment we conclude that both energy consumption and performance are better whether the large number of VMs. For future work it has still limitation when number of VMs close to maximum. In future we will try to improve this limitation.

\section{References}

[1]. V.Krishna Ready, B.Thirumala Rao, Dr.L.S.S.Ready, P.Sai Kiran" Research Issues in Cloud Computing"Global Journal of Computer Science and Technology, Volume 11 Issue 11 Version 1.0 July 2011.

[2]. M. Armbrust, A. Fox, R. Griffith, A. D. Joseph, R. Katz, A. Konwinski, G. Lee, D. Patterson, A. Rabkin, I. Stoica, and M. Zaharia. "Above the Clouds: A Berkeley View of Cloud Computing," Technical Report EECS-2009-28, EECS Department, Univ.of California, Berkeley, 2009.

[3]. Mohiuddin Ahmed, Abu Sina Md. Raju Chowdhury, “An Adavanced Survey On Cloud Computing and State-of-art Research Issues" IJCSI International Journal of Computer Science Issues, Vol.9, Issue 1,January 2012 ISSN(Online): 1694-0814

[4]. Hamilton J "Cooperative expendable micro-slice servers (CEMS): low cost, low power servers for Internet-scale services In: Proc of CIDR",(2009)

[5]. A. Beloglazov, R. Buyya, Y. C. Lee, "A taxonomy and survey of energy-efficient data centers and Cloud computing systems" Advances in Computers, M. Zelkowitz (ed.), vol. 82, pp. 47-111, 2011.

[6]. "Growth in data center electricity use 2005 to 2010" Analytics Press, Tech. Rep., 2011.

[7]. Li B et al (2009) "EnaCloud: an energy-saving application live placement approach for cloud computing environments". In: Proc of international conf on cloud computing.

[8]. K. H. Kim, A. Beloglazov and R. Buyya. "Power-aware provisioning of virtual machines for real-time Cloud services," Concurrency and Computation: Practice \& Experience archive Volume 23 Issue 13, pp. 1491-1505 2011.

[9]. R. N. Calheiros, R. Ranjan, A. Beloglazov, Cesar A. F. De Rose, R. Buyya. "CloudSim: A Toolkit for Modeling and Simulation of Cloud Computing Environments and Evaluation of Resource Provisioning Algorithms," Software: Practice and Experience (SPE), pp. 23-50, 2011.

[10]. Buyya R, Yeo CS, Venugopal S, Broberg J, Brandic I. "Cloud computing and emerging IT platforms: vision, hype, and reality for delivering computing as the $\mathbf{5}^{\text {th }}$ utility. Future Generation Computer Systems". 25(6):599-616, Elsevier Science, Amsterdam, The Netherlands, June 2009.

[11]. Pouwelse, J., Langendoen, K., Sips, H.: "Dynamic Voltage on a Low-Power Microprocessor System". In: Proceedings of the International Conference on Mobile Computing and Networking, pp. 251-259 (2001)

[12]. Bo Li ; Shuaiwen Song ; Bezakova, I. ; Cameron, K.W. "Energy-Aware Replica Selection for Data-Intensive Services in Cloud" Modeling, Analysis \& Simulation of Computer and Telecommunication Systems (MASCOTS), 2012 IEEE 20th International Symposium on Digital Object Identifier: 10.1109/MASCOTS.2012.66 Publication Year: 2012, Page(s): 504 - 506.

[13]. Chawarut, W. ; Woraphon, L. "Energy-aware and real-time service management in cloud computing" Electrical Engineering/Electronics, Computer, Telecommunications and Information Technology(ECTI-CON), 2013 10th International Conference on Digital Object Identifier:10.1109/ECTICon.2013.6559495 Publication Year: 2013, Page(s): 1 - 5.

[14]. Li, Y.F., Li, W., Jiang, C.F.: "A Survey of Virtual Machine System: Current Technology and Future Trends."In: Third International Symposium on Electronic Commerce andSecurity, pp. 332-336 (2010)

[15]. K. H. Kim, A. Beloglazov and R. Buyya. "Power-aware provisioning of virtual machines for real-time Cloud services," Concurrency and Computation: Practice \& Experience archive Volume 23 Issue 13, pp. 1491-1505 2011.

[16]. Liang-Teh Lee, Kang-Yuan Liu, Hui-Yang Huang and Chia-Ying Tseng "A Dynamic Resource Management with Energy Saving Mechanism for Supporting Cloud Computing” Department of Computer Science and Engineering, Tatung University, Taiwan.

[17]. J. Baliga, K. Hinton, and R. S. Tucker, "Energy consumption of the Internet" The International Conference on the Optical Internet with the $32^{n d}$ Australian Conference on Optical Fibre Technology, 2007, pp.1-3. 
[18]. N. Rodrigo, Calheiros, R. Ranjan, A. Beloglazov, C. A. F. D. Rose and R. Buyya, "CloudSim: A Toolkit for Modeling and Simulation of Cloud Computing Environments and Evaluation of Resource Provisioning Algorithms", ISSN:0038-0644, Wiley Press, New York, USA (2011), pp. 23-50.

[19]. K. H. Kim, A. Beloglazov and R. Buyya. "Power-aware provisioning of virtual machines for real-time Cloud services," Concurrency and Computation: Practice \& Experience archive Volume 23 Issue 13, pp. 1491-1505 2011.

[20]. Li, Y.F., Li, W., Jiang, C.F.: "A Survey of Virtual Machine System: Current Technology and Future Trends."In: Third International Symposium on Electronic Commerce andSecurity, pp. 332-336 (2010) 